\title{
A Modified Method for Solving Delay Differential Equations of Fractional Order
}

\author{
Osama Hameed Mohammed, Qutaiba Wadi \\ ${ }^{1,2}$ Al-Nahrain University, College of Science, Department of Mathematics.
}

\begin{abstract}
In this paper, generalized Hat functions operational matricesare proposed and combined with the method of steps to solve linear and nonlinear delay differential equations of fractional order. We convert the delay differential equationsof fractional order to non-delay differential equationsof fractional orderon a given intervalby apply the method of steps, and then apply the operational matrices for generalized Hat function on the obtained non-delay differential equationsof fractional order to transform linear and nonlinear non-delay differential equationsof fractional order into a system of algebraic equations and then find the solution.Two illustrative examples will be presented to show the accuracy and efficiencyof the proposed method.
\end{abstract}

Keywords: Fractional calculus, Delay differential equations, Fractional delay differential equations, Hat functions, Method of Steps.

\section{Introduction}

Fractional calculus (that is, calculus of integrals and derivatives of any arbitrary real or complex order) is an old mathematical problem, and mainly developed as a pure mathematics problem for nearly three centuries [1]-[3]. Though having a long history, it was not used in physics and engineering for a long period. However, in the last few decades, fractional calculus began to attract increasing attention of scientists from the viewpoint of application [3]-[6]. Fractional calculus and fractional differential equations have found applications in several different disciplines [7]-[8].

Over the years, many mathematicians, using their own notation and approach, have found various definitions that fit the idea of a non-integer order integral or derivative. The most famous of these definitions that have been popularized in the world of fractional calculus are Riemann-Liouville and Grünwald-Letnikov definition. Also, Caputo, [9] reformulated the more "classic" definition of the Riemann-Liouville fractional derivative in order to use integer order initial conditions to solve his fractional order differential equations.

Delay differential equations (DDEs) are a type of differential equation in which the derivative of the unknown function at a certain time is given in terms of the values of the function at previous times. Delay differential equations play an important role in the research field of various applied sciences such as control theory, electrical networks, population dynamics, environment science, biology and life science [10].

Fractional delay differential equations are a very recent topic. Although it seems natural to model certain processes and systems in engineering and other sciences with this kind of equations, only in the last few years has the attention of the scientific community been devoted to them [11], [12], [13].

This paper is organized as follows: In section 2 we recall the definitions of fractional derivatives and fractional integration, in section 3, a review of generalized Hat functions and their properties is described. In section 4, the operational matrices of integration for generalized Hat functions is derived. In section 5, the proposed method is described. In section 6, some illustrative examples are presented. Finally, a conclusion is drawn in section 7 .

\section{Fractional Integral And Differential Operators}

In this section, we review basic definitions of fractionaldifferentiation and fractional integration [3].

Definition2.1: The Riemann-Liouville fractional order integral operator $I_{t}^{\alpha}$ of order $\quad \alpha \geq 0$, of a function $u(t) \in L_{2}[a, b]$ is given by: -

$I_{t}^{\alpha} u(t)= \begin{cases}\frac{1}{\Gamma(\alpha)} \int_{0}^{t}(t-s)^{\alpha-1} u(s) d s, & \alpha>0, \\ u(t), & \alpha=0,\end{cases}$

where $a \leq t \leq b$.

Definition 2.2:The Riemann-Liouville fractional order derivatives operator $D_{t}^{\alpha}$ of order $\alpha \geq 0$, of a function $u(t) \in L_{2}[a, b]$ is given by: - 
$D_{t}^{\alpha} u(t)=\frac{1}{\Gamma(n-\alpha)}\left(\frac{d}{d t}\right)^{n} \int_{0}^{t}(t-s)^{n-\alpha-1} u(s) d s .(2)$

for $a \leq t \leq b$, where $\alpha \in R^{+}$and $n$ is integer.

The Riemann-Liouville derivatives have certain disadvantages when trying to model real-world phenomena with fractional differential equations. Therefore, we shall introduce a modified fractional differential operator ${ }^{c} D_{t}^{\alpha}$ which is proposed by Caputo [9].

Definition 2.3: The Caputo fractional derivative of a function $u(t) \in L_{1}[\mathrm{a}, \mathrm{b}]$ is given by:-

${ }^{c} D_{t}^{\alpha} u(t)=\frac{1}{\Gamma(n-\alpha)} \int_{0}^{t}(t-s)^{n-\alpha-1}\left(\frac{d}{d t}\right)^{n} u(s) d s$.(3)

for $a \leq t \leq b$, where $\alpha \in R^{+}$and $n$ is integer.

For $f(t) \in C^{m}[a, b], \alpha, \beta \geq 0, n-1<\alpha \leq n, \alpha+\beta \leq m, v \geq-1$, the fractional integral and derivatives satisfy the following:

1. $\left(I_{t}^{\alpha} I_{t}^{\beta} u\right)(t)=\left(I_{t}^{\beta} I_{t}^{\alpha} u\right)(t)=\left(I_{t}^{\alpha+\beta} u\right)(t)$.

2. $\left(I_{t}^{\alpha}{ }^{c} D^{\alpha} u\right)(t)=u(t)-\sum_{k=0}^{n-1} u^{(k)}\left(0^{+}\right) \frac{t^{k}}{k !}$.

3. $\left(I_{t}^{\alpha} t^{v}\right)=\frac{\Gamma(v+1)}{\Gamma(v+\alpha+1)} t^{\alpha+v}$.

III. Generalized Hat Functions And Their Properties [14]

The traditional Hat functions are continuous functions, also called triangle, tent or triangular functions are defined on the interval $[0,1]$.The generalized Hat functions are extension of traditional Hat functions on the finite interval $[0, A]$. The interval $[0, A]$ is divided into $\mathrm{n}$ equidistant subintervals, $[\mathrm{ih},(\mathrm{i}+1) \mathrm{h}]$ of equal lengths $\mathrm{h}$ where $\mathrm{h}=\mathrm{A} / \mathrm{n}$ and nis an arbitrary positive integer. The generalized Hat function's family of first $(\mathrm{n}+1)$ Hat functions are usually defined on $[0, \mathrm{~A}]$ as $[15]$ :

$\varphi_{0}(t)=\left\{\begin{array}{l}\frac{h-t}{h}, 0 \leq t<h \\ 0, \text { otherwise }\end{array}\right.$

$\varphi_{i}(t)=\left\{\begin{array}{l}\frac{t-(i-1) h}{h},(i-1) h \leq t<h \\ \frac{(i+1) h-t}{h}, i h \leq t<(i+1) h . i=1,2, \ldots, n-1 \\ 0, \text { otherwise }\end{array}\right.$

$\varphi_{n}(t)=\left\{\begin{array}{l}\frac{t-(A-h)}{h}, A-h \leq t \leq A \\ 0, \text { otherwise }\end{array}\right.$

According to the definition of Hat functions:

$\varphi_{i}(j h)= \begin{cases}1, & i=j \\ 0, & i \neq j\end{cases}$

And $\quad \varphi_{i}(t) \varphi_{j}(t)=0, \quad|i-j| \geq 2$.

And $\sum_{i=0}^{n} \varphi_{i}(\mathrm{t})=1$. 


\subsection{Function Approximation}

An arbitrary function $g \in L^{2}[a, b]$ is approximated in vector form as:

$g(t)=\sum_{i=0}^{n} f_{i} \varphi_{i}(t)=G_{n+1}^{T} \Phi_{n+1}(t)=\Phi_{n+1}^{T}(t) G_{n+1}$,

where

$G_{n+1} \triangleq\left[g_{0}, g_{1}, g_{2}, \ldots, g_{n}\right]^{T}$,

and

$\Phi_{n+1}(t) \triangleq\left[\varphi_{0}(t), \varphi_{1}(t), \varphi_{2}(t), \ldots, \varphi_{n}(t)\right]^{T}$.

The important aspect of using generalized Hat functions in theapproximation of function $g(t)$, lies in the fact that the coefficients $g_{i}$ in the Eq. (9), are given by

$g_{i}=g(i h), \quad i=0,1,2, \ldots, n$.

\section{Operational Matrices of The Integration for Generalized Hat Functions}

The integer order and fractional order operational matrices of integration for generalized Hat functions is given in the subsections (4.1) and (4.2) respectively.

\subsection{Integer Order Operational Matrix of Integration of the Generalized Hat Functions}

Since $\int_{0}^{t} \varphi_{i}(\tau) d \tau \in L_{2}[0, A]$,Eq. (10) is used to approximate it in the terms of the generalized Hat basis functions as

$\int_{0}^{t} \varphi_{i}(\tau) d \tau \simeq \sum_{j=0}^{n} b_{i j} \varphi_{j}(t), \quad i=0,1,2, \ldots, n$.

Using Eq. (11), we calculate the coefficients $a_{i j}$ as

$b_{i j}=\int_{0}^{i h} \varphi_{i}(\tau) d \tau, \quad j=0,1,2, \ldots, n$.

The coefficients $b_{i j}$ will form a $(n+1) \times(n+1)$ matrix $P_{n+1}$ with $(i+1, j+1)^{t h}$ entry as $b_{i j}$, for $i=$ $0,1,2, \ldots, n, j=0,1,2, \ldots, n$.Using the values of $b_{i j}$ 's from Eq. (12), we obtain the matrix $P_{n+1}$ as:

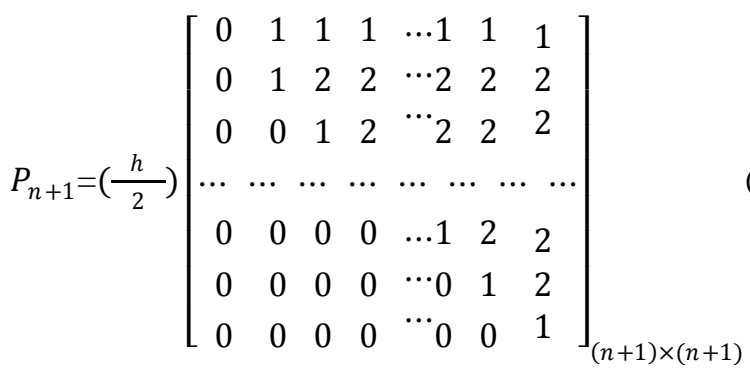

The matrix $P_{n+1}$ is called the integer order Hat functions operational matrix of integration.

It plays a pivotal role in determination of $\int_{0}^{t} g(\tau) d \tau$ for an arbitrary $g \in L_{2}[0, A]$. with the help of Eqs. (10) and (11), we have

$\int_{0}^{t} \varphi_{n+1}(\tau) d \tau=P_{n+1} \Phi_{n+1}(t)$.

4.2Fractional orderOperational Matrix of Integration of the Generalized Hat Functions The fractional integration of generalized Hat function in Eq. (10) can be approximated as

$\left(I_{t}^{\alpha} \Phi_{n+1}\right)(t)=P_{n+1}^{\alpha} \Phi_{n+1}(t)$.

where

$P_{n+1}^{\alpha}=\frac{h^{\alpha}}{\Gamma(\alpha+2)}\left[\begin{array}{ccccc}0 & \gamma_{1} & \gamma_{2} & & \gamma_{n} \\ 0 & 1 & \zeta_{1} & \cdots & \zeta_{n-1} \\ 0 & 0 & 1 & & \zeta_{n-2} \\ & \vdots & & \ddots & \vdots \\ 0 & 0 & 0 & \cdots & 1\end{array}\right]_{(n+1) \times(n+1)}$

where

$$
\gamma_{k}=k^{\alpha}(\alpha-k+1)+(k-1)^{\alpha+1}, k=1,2, \ldots, n .
$$

and

$\zeta_{k}=(k+1)^{\alpha+1}-2 k^{\alpha+1}+(k-1)^{\alpha+1}, k=1,2, \ldots, n-1$.

For more details,one can see[15]. 


\section{The Approach}

In this section, we shall approximate solution of the following fractional order delay differential equations:

${ }^{c} D_{t}^{\alpha} u(t)=F(t, u(t), u(\phi(t))), n-1<\alpha \leq n, t>0$

$u(t)=\psi(t), t \in[-\tau, 0]$

$u^{(i)}(0)=u_{0}^{(i)}, i=0,1,2, \ldots, n-1$

where ${ }^{c} D_{t}^{\alpha}$ isCaputo fractional derivative of order $\alpha, F$ is a nonlinear operator, $t$ is the independent variable, $u(t)$ is the unknown function, $\phi(t)$ is the delay function $\psi(t)$ is given functions and $u^{(i)}(0)$ are given constants.

First we convert the fractional order delay differential equation to fractional order non-delay differential equation by applying the method of steps [13], as

${ }^{c} D_{t}^{\alpha} u(t)=F(t, u(t), \psi(\phi(t))), n-1<\alpha \leq n, t>0$

$u^{(i)}(0)=u_{0}^{(i)}, i=0,1,2, \ldots, n-1$

Now in order to solve Eqs. (17)- (18) by using the operational matrices of generalized Hat functions, we approximate ${ }^{c} D_{t}^{\alpha} u(t)$ and $u(t)$ in terms of generalized Hat functions as follows

$\left({ }^{c} D_{t}^{\alpha} u\right)(t)=C_{n+1}^{T} \Phi_{n+1}(t)(19)$

And upon operating $I_{t}^{\alpha}$ to the both sides of equation (19) leads to

$u(t)=C_{n+1}^{T} P_{n+1}^{\alpha} \Phi_{n+1}(t)+\sum_{k=0}^{n-1} u^{(k)}\left(0^{+}\right) \frac{t^{k}}{k !}$

where

$\Phi_{n+1}(t) \triangleq\left[\varphi_{0}(t), \varphi_{1}(\mathrm{t}), \varphi_{2}(t), \ldots, \varphi_{n}(t)\right]^{T}$,

and

$C_{n+1}(t) \triangleq\left[c_{0}, c_{1}, c_{2}, \ldots, c_{n}\right]^{T}$.

Hence

$F(t, u(t), u(\phi(t)))=F\left(t, C_{n+1}^{T} P_{n+1}^{\alpha} \Psi_{n+1}(t)+\sum_{k=0}^{n-1} u^{(k)}\left(0^{+}\right) \frac{t^{k}}{k !}, \psi(\phi(t))\right)$

Substituting Eqs. (19) and (21) into Eq. (17) gives

$C_{n+1}^{T} \Phi_{n+1}(t)=F\left(t, C_{n+1}^{T} P_{n+1}^{\alpha} \Phi_{n+1}(t)+\sum_{k=0}^{n-1} u^{(k)}\left(0^{+}\right) \frac{t^{k}}{k !}, \psi(\phi(t))\right)(22)$

Also, by substituting Eqs. (11) and (19) into Eq. (18), we get

$u^{(i)}(0)=C_{n+1}^{T} \Phi_{n+1}(0)=u_{0}^{(i)}, i=0,1,2, \ldots, n-1$

From Eq. (22), we can obtain the coefficients $C_{n+1}^{T}$. Then using Eq.(20), we can get the output response $u(t)$.

\section{Illustrative Examples}

In this section, we shall solve linear and nonlinear delay differential equations of fractional order by using the approach given in section 5, and compare the results that we have been obtained with the existing methods and the exact solution. we refer $u_{\text {hat }}$ to represent the solution by generalized Hat functions, $u_{c h}$ to represent the solution by Chebyshev wavelets method and $u_{\text {exact }}$ to represent the exact solution.

\section{Example (1):}

Consider the delay differential equations of fractional order with nonlinear delay function

${ }^{c} D_{t}^{\alpha} u(t)=1-2 u^{2}\left(\frac{t}{2}\right), \quad 0<\alpha \leq 1,0<t \leq 1$

$u(t)=\sin (t),-1 \leq t \leq 0$

$u(0)=0(26)$

The exact solution, when $\alpha=1$, is $u(t)=\sin (t)$.

\section{Solution:}

First we convert the delay differential equation of fractional order to non-delay differential equation of fractional order by applying the method of steps, as

$$
{ }^{c} D_{t}^{\alpha} u(t)=1-2 \sin ^{2}\left(\frac{t}{2}\right) .0<\alpha \leq 1.0<t \leq 1
$$

$u(0)=0$

Now we approximate ${ }^{c} D_{t}^{\alpha} u(t)$ in Eq. (27), in terms of generalized Hat functions as follows

$\left({ }^{c} D_{t}^{\alpha} u\right)(t)=C_{n+1}^{T} \Phi_{n+1}(t)(29)$

Hence 
$u(t)=C_{n+1}^{T} P_{n+1}^{\alpha} \Phi_{n+1}(t) \quad(30)$

Also writing the term $1-2 \sin ^{2}\left(\frac{t}{2}\right)$ in Eq. (27) in terms of generalized Hat functions leads to

$1-2 \sin ^{2}\left(\frac{t}{2}\right)=G_{n+1} \Phi_{n+1}(t)$,

Where

$G_{n+1} \triangleq\left[g_{0}, g_{1}, g_{2}, \ldots, g_{n}\right]^{T}$,

and

$g_{i}=1-2 \sin ^{2}\left(\frac{i h}{2}\right), \quad i=0,1,2, \ldots, n$.

Substituting Eqs. (29) and (31) into Eq. (27), we have

$C_{n+1}^{T} \Phi_{n+1}(t)=G_{n+1} \Phi_{n+1}(t), \quad(32)$

which implies that

$C_{n+1}^{T}=G_{n+1}^{T}$

Solving Eq. (33), we can obtain the coefficients $C_{n+1}^{T}$. Then using Eq.(30), one can get the output response $u(t)$.

For $n=8$, it seems from Table (1) that the results obtained from the proposed method when $\alpha=1$ provides better results as compared with the existing methods such as Chebyshev wavelet method and the exact solution.

Table 1: Comparison of The Approximate Solution of Example (1) Using The Proposed Method And Chebyshev Wavelet Method When $\alpha=1$ And The Exact Solution.

\begin{tabular}{|l|l|l|l|}
\hline$T$ & & & \\
\hline $\mathbf{0}$ & 0 & 0 & 0 \\
\hline $\mathbf{0 . 1 2 5}$ & 0.124 & 0.124 & 0.124 \\
\hline $\mathbf{0 . 2 5 0}$ & 0.246 & 0.247 & 0.247 \\
\hline $\mathbf{0 . 3 7 5}$ & 0.355 & 0.365 & 0.366 \\
\hline $\mathbf{0 . 5 0 0}$ & 0.464 & 0.478 & 0.479 \\
\hline $\mathbf{0 . 6 2 5}$ & 0.581 & 0.584 & 0.585 \\
\hline $\mathbf{0 . 7 5 0}$ & 0.682 & 0.680 & 0.681 \\
\hline $\mathbf{0 . 8 7 5}$ & 0.755 & 0.766 & 0.767 \\
\hline $\mathbf{1}$ & 0.846 & 0.840 & 0.841 \\
\hline
\end{tabular}

Following Figure (1) represent the approximate solution of example (1) using the proposed method for different values of $\alpha$ and the exact solution when $\alpha=1$.

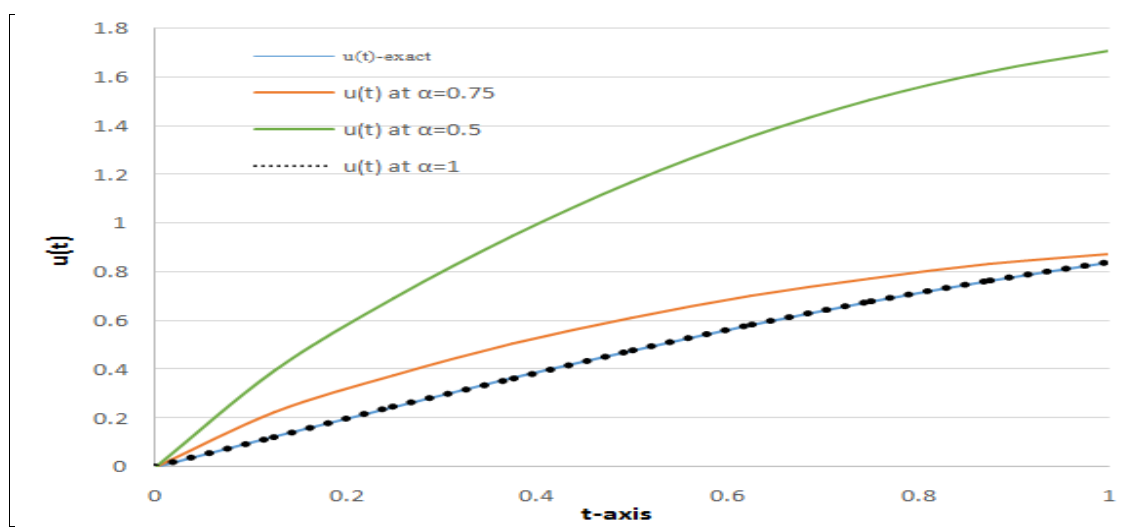

Fig. 1: The approximate solution of example (1) by using the proposed method at different values of $\alpha$ and the exact solutions at $\alpha=1$.

Example (2):

Consider the delay differential equation of fractional order

$$
{ }^{c} D_{t}^{\alpha} u(t)-u\left(\frac{t}{2}\right)=0, \quad 0<\alpha \leq 1, \quad 0<t \leq 1
$$

$u(t)=1+\mathrm{t},-1 \leq t \leq 0$

$u(0)=1(36)$

The exact solution is $u(t)=\sum_{k=0}^{\infty} \frac{\left(\frac{1}{2}\right)^{\frac{1}{2} k(k-1)}}{k !} t^{k}$.

\section{Solution:}

First we convert the delay differential equation of frractional order tonon-delay differential equation of fractional order by applying the method of steps, as: 
${ }^{c} D_{t}^{\alpha} u(t)=1+\frac{t}{2}, 0<\alpha \leq 1,0<t \leq 1$

$u(0)=1$

Now we approximate ${ }^{c} D_{t}^{\alpha} u(t)$ in Eq. (37), in terms of generalized Hat functions as follows

$\left({ }^{c} D_{t}^{\alpha} u\right)(t)=C_{n+1}^{T} \Phi_{n+1}(t)$

Hence

$u(t)=C_{n+1}^{T} P_{n+1}^{\alpha} \Phi_{n+1}(t)+1$

Also writing the term $1+\frac{t}{2}$ in Eq. (37) in terms of generalized Hat functions leads to

$1+\frac{t}{2}=G_{n+1} \Phi_{n+1}(t)$,

Where

$G_{n+1} \triangleq\left[g_{0}, g_{1}, g_{2}, \ldots, g_{n}\right]^{T}$,

and

$g_{i}=1+\frac{i h}{2}, \quad i=0,1,2, \ldots, n$.

Substituting Eqs. (39) and (41) into Eq. (37), we have

$C_{n+1}^{T} \Phi_{n+1}(t)=G_{n+1} \Phi_{n+1}(t)$,

which implies that

$C_{n+1}^{T}=G_{n+1}^{T} \quad$ (43)

Then using Eq.(40), one can get the output response $u(t)$.

For $n=8$, it seems from Table (2) that the results obtained from the proposed method when $\alpha=1$ provides better results as compared with the existing methods such as Chebyshev wavelet method and the exact solution.

Table 2: Comparison of The Approximate Solution of Example (2) Using The Proposed Method And Chebyshev Wavelet Method When $\alpha=1$ And The Exact Solution.

\begin{tabular}{|l|l|l|l|}
\hline $\mathrm{t}$ & $\boldsymbol{\alpha}=1$ & \multicolumn{1}{c|}{$\begin{array}{c}\boldsymbol{u}_{\text {hat }} \\
\boldsymbol{\alpha}=\mathbf{1}\end{array}$} & $\begin{array}{c}\boldsymbol{u}_{\text {exact }} \\
\boldsymbol{\alpha}=\mathbf{1}\end{array}$ \\
\hline $\mathbf{0}$ & 1 & 1 & 1 \\
\hline $\mathbf{0 . 1 2 5}$ & 1.13 & 1.13 & 1.12 \\
\hline $\mathbf{0 . 2 5 0}$ & 1.28 & 1.26 & 1.26 \\
\hline $\mathbf{0 . 3 7 5}$ & 1.44 & 1.41 & 1.41 \\
\hline $\mathbf{0 . 5 0 0}$ & 1.62 & 1.56 & 1.56 \\
\hline $\mathbf{0 . 6 2 5}$ & 1.82 & 1.72 & 1.72 \\
\hline $\mathbf{0 . 7 5 0}$ & 2.03 & 1.89 & 1.90 \\
\hline $\mathbf{0 . 8 7 5}$ & 2.26 & 2.06 & 2.08 \\
\hline $\mathbf{1}$ & 2.50 & 2.25 & 2.27 \\
\hline
\end{tabular}

Following Figure (2)represent the approximate solution of example (2) using the proposed method for different values of $\alpha$ and the exact solution when $\alpha=1$.

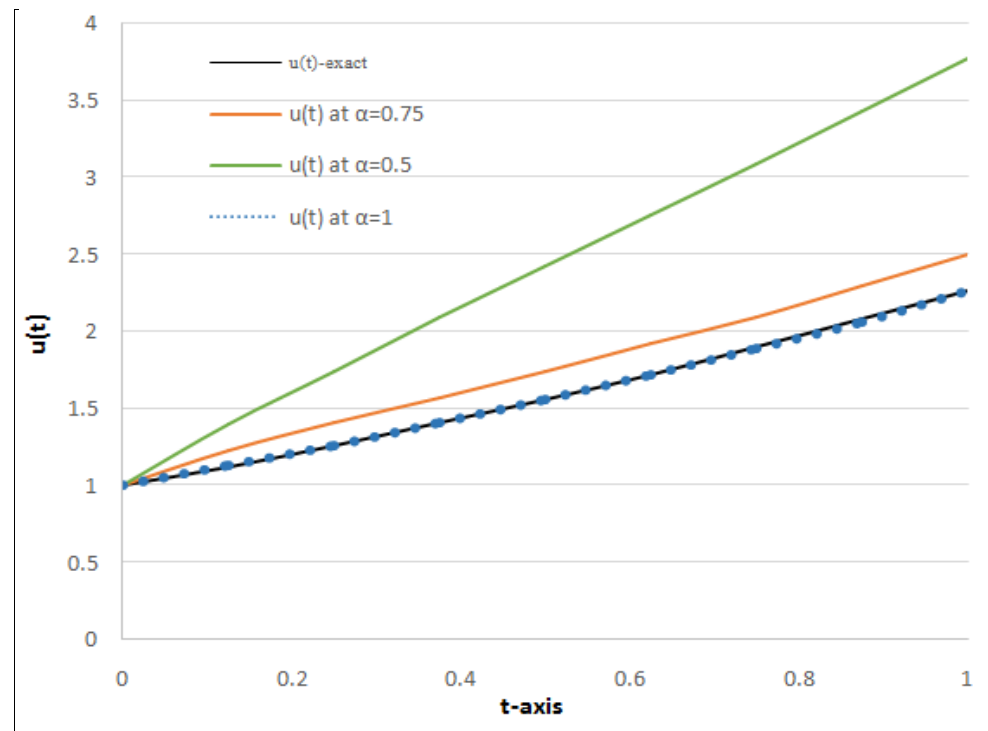

Fig. 2: The approximate solution of example (2) by using the proposed method at different values of $\alpha$ and the exact solutions at $\alpha=1$. 


\section{Conclusion}

In this paper, wepresent the integer and fractional orders of integration for the generalized hat functions operational matrices and combined them with the method of steps to solve linear and nonlinear delay differential equations of fractional order numerically. The obtained results are compared with the exact solutions and with the solutions obtained by some other numerical methods such as Chebyshev wavelet method. The results obtained from the proposed methodare more accurate and better than the results obtained from Chebyshev wavelet methodand are in good agreement with the exact solution.

\section{References}

[1] Loverro, A., “Fractional Calculus: History, Definitions and Applications for the Engineer", Department of Aerospace and Mechanical Engineering, University of Notre Dame, Notre Dame, IN 46556, USA, May 8, 2004.

[2] KolowankarK. M. and Gangal, "Fractional Differentiability of no-where differentiable functions and dimensions", CHAOS V.6, No.4, American Institute of Physics, 1996.

[3] Podlubny I. "“Fractional Differential Equations ”, Mathematics in Science and Engineering V198, Academic Press, 1999.

[4] Wang Z., Huang X., Li N. and Song X., Chin. Phys. B, 5 050506(2012).

[5] KilbasA. A., Srivastava H. M. and Trujillo J. J. , Theory and Applications of Fractional Di fferential Equations. Elsevier, Amsterdam (2006).

[6] HilferR., Applications of Fractional Calculus in Physics, Hackensack, NJ: World Scientific, 2001.

[7] He JH. Nonlinear oscillation with fractional derivative and its applications. Int ConfVibrEng 1998:288e91.

[8] He JH. Some applications of nonlinearfractional differential equations and their approximations. Bull Sci Tech 1999;15(2):86e90.

[9] MoragdoM.L., Ford N. J. and Lima P.M., 'Analysisand numerical methods for fractional differential equations with delay", Journal of computational and applied mathematics 252(2013) 159-168.

[10] Smith H., An Introduction to Delay Differential Equations with Applications to the Life Sciences, Springer, New York, NY, USA, 2011.

[11] LuchkoA.GrorefloY.,R.,The initial value problem for some fractional differential equations with the Caputo derivative, Preprint series A 08-98, Fachbreich Mathematik und Informatik, FreicUniversitat Berlin, 1998.

[12] B.P. Moghaddam, Z.S. Mostaghim, A numerical method based on finite difference for solving fractional delay differential equations, J. Taibah Univ. Sci.7 (2013) 120-127.

[13] M.L. Morgado, N.J.Ford, P.M.Lima, Analysis and numerical methods for fractional differential equations with delay, J. Comput. Appl. Math. 252 (2013) 159-168.

[14] BabolianE., Mordad M., A numerical method for solving systems of linear and nonlinear integral equations of the second kind by hat basis functions, Comput. Math. Appl. 62 (1) (2011) 187-198.

[15] Tripathi M. P., Baranwal V. K., Pandey R. K., and Singh O. P., A new numerical algorithm to solve fractional differential equations based on operational matrix of generalized Hat functions, Commun Nonlinear SciNumerSimulat, vol. 18, pp. 1327 1340,2013. 